\title{
Bioinspired polydopamine (PDA) chemistry meets ordered mesoporous carbons (OMCs): a benign surface modification strategy for versatile functionalization
}

Yang Song ${ }^{\dagger \|}$, Gang Ye* ${ }^{\star \dagger}$, Fengcheng $\mathrm{Wu}^{\dagger}$, Zhe Wang ${ }^{\dagger}$, Siyuan Liu ${ }^{\S}$, Maciej

Kopeć", Zongyu Wang", Jing Chen ${ }^{\dagger \ddagger}$, Jianchen Wang ${ }^{\star \dagger \ddagger}$, Krzysztof

Matyjaszewski*"

${ }^{\dagger}$ Collaborative Innovation Center of Advanced Nuclear Energy Technology, Institute of Nuclear and New Energy Technology, Tsinghua University, Beijing, 100084, China

${ }^{\ddagger}$ Beijing Key Lab of Radioactive Waste Treatment, Tsinghua University, Beijing 100084, China

$\S$ Department of Materials Science and Engineering, Carnegie Mellon University,

" Department of Chemistry, Carnegie Mellon University, 440o Fifth Avenue, Pittsburgh,

Pennsylvania 15213, United States 


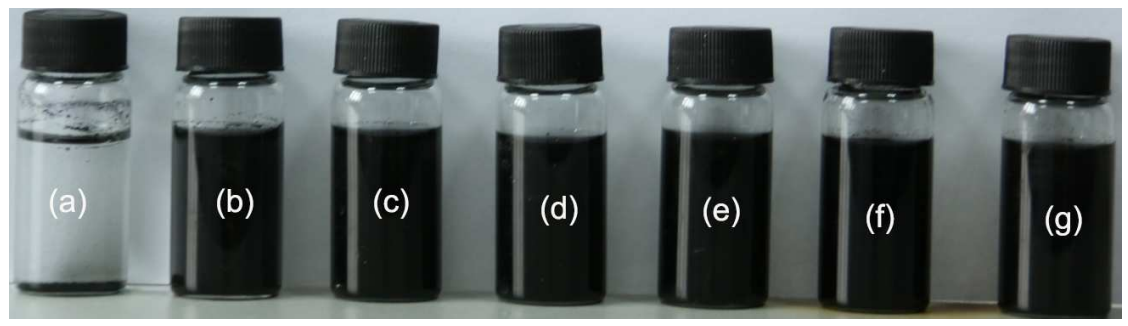

Figure S1. Water dispersibility of (a) CMK-3; (b) CMK-3-PDA-0.6-10; (c) CMK-3-PDA-1.1-10; (d) CMK-3-PDA-2.2-10; $\quad$ (e) CMK-3-PDA-4.4-10; $\quad$ (f) CMK-3-PDA-1.1-5; (g) CMK-3-PDA-1.1-24. 


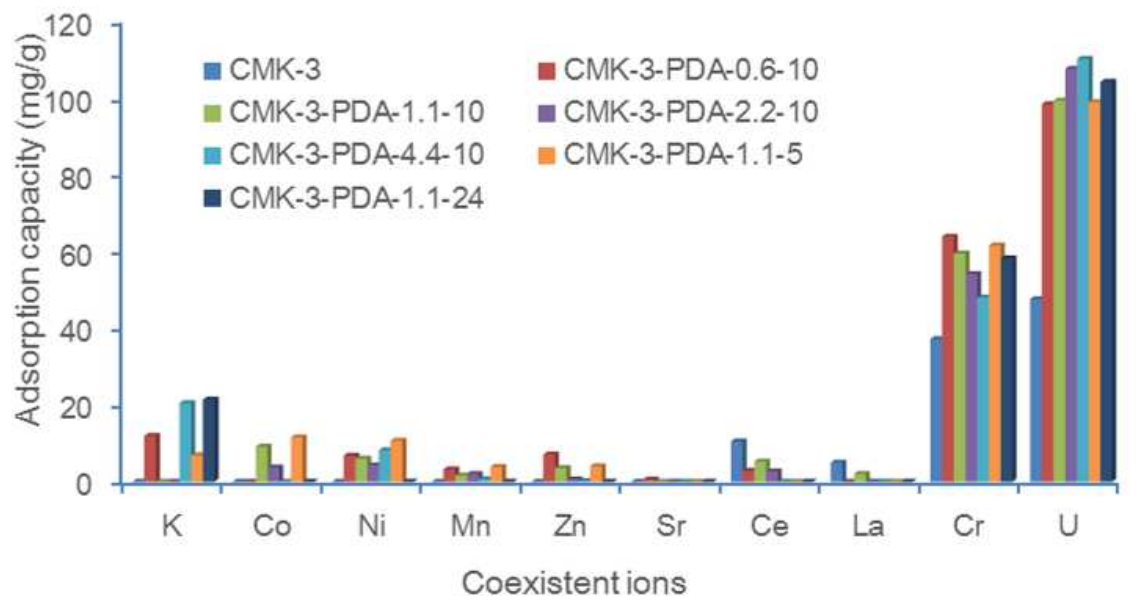

Figure S2. Competitive adsorption of U(VI) by CMK-3 and PDA-coated CMK-3 against coexistent metal ions in a simulated nuclear industry effluent $(\mathrm{pH}=5$, contact time $=24 \mathrm{~h}$ ). 


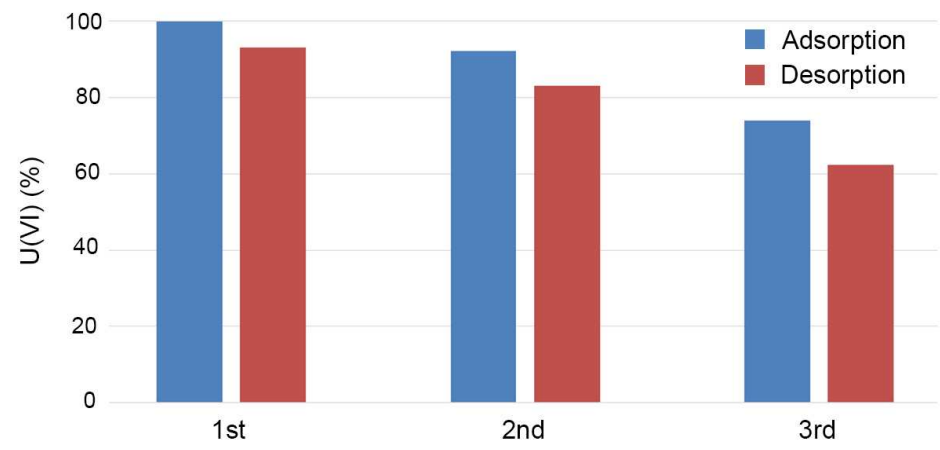

Figure S3. Adsorption and desorption efficiency of U(VI) by CMK-3-PDA-4-4-24 in cycle use. Initial $\mathrm{U}(\mathrm{VI})$ concentration $=50 \mathrm{mg} / \mathrm{L}, \mathrm{pH}=5, \mathrm{~T}=301 \mathrm{~K}$, contact time for either adsorption or desorption $=24 \mathrm{~h}$. Data were normalized by setting the adsorption efficiency in the $\mathrm{1}^{\text {st }}$ run to be $100 \%$. 


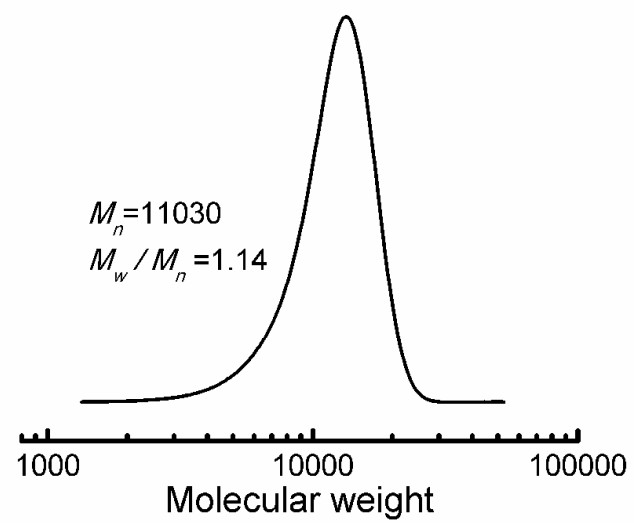

Figure S4. Molecular weight distribution of PMMA ([MMA]: [EBiB]: [ $\left.\mathrm{CuBr}_{2}\right]$ : [TPMA]: $\left[\mathrm{Sn}(\mathrm{EH})_{2}\right]=70: 1: 0.0035: 0.035: 0.0175$, solvent: anisole, $\left.24 \mathrm{~h}, 25^{\circ} \mathrm{C}\right)$. 
Table S1. Composition of the simulated nuclear industry effluent.

\begin{tabular}{ccc}
\hline Coexistent ions & Metal salts & Concentration $(\mathrm{mg} / \mathrm{L})$ \\
\hline $\mathrm{K}^{+}$ & $\mathrm{KCl}$ & 295.8 \\
$\mathrm{Co}^{2+}$ & $\mathrm{CoCl}_{2} \cdot 6 \mathrm{H}_{2} \mathrm{O}$ & 126.8 \\
$\mathrm{Ni}^{2+}$ & $\mathrm{Ni}\left(\mathrm{NO}_{3}\right)_{2} \cdot 6 \mathrm{H}_{2} \mathrm{O}$ & 161.1 \\
$\mathrm{Mn}^{2+}$ & $\mathrm{MnCl}_{2} \cdot 4 \mathrm{H}_{2} \mathrm{O}$ & 161.7 \\
$\mathrm{Zn}^{2+}$ & $\mathrm{ZnCl}_{2}$ & 133.5 \\
$\mathrm{Sr}^{2+}$ & $\mathrm{SrCl}_{2} \cdot 6 \mathrm{H}_{2} \mathrm{O}$ & 209.3 \\
$\mathrm{Ce}^{3+}$ & $\mathrm{Ce}\left(\mathrm{NO}_{3}\right)_{3} \cdot 6 \mathrm{H}_{2} \mathrm{O}$ & 259.6 \\
$\mathrm{La}^{3+}$ & $\mathrm{LaCl}_{3} \cdot \mathrm{nH}_{2} \mathrm{O}$ & 274.6 \\
$\mathrm{Cr}^{3+}$ & $\mathrm{CrCl}_{3} \cdot 6 \mathrm{H}_{2} \mathrm{O}$ & 99.5 \\
$\mathrm{UO}_{2}^{2+}$ & $\mathrm{U}_{3} \mathrm{O}_{8}$ & 180.8 \\
\hline
\end{tabular}


Table S2. Structural parameters of pristine CMK-3 and the PDA coated CMK-3 adsorbents.

\begin{tabular}{cccc}
\hline Samples & $\begin{array}{c}\text { surface area } \\
\left(\mathrm{m}^{2} / \mathrm{g}\right)\end{array}$ & $\begin{array}{c}\text { pore volume } \\
\left(\mathrm{m}^{3} / \mathrm{g}\right)\end{array}$ & $\begin{array}{c}\text { pore size } \\
(\mathrm{nm})\end{array}$ \\
\hline CMK-3 & 1114 & 1.28 & 3.63 \\
CMK-3-PDA-0.6-10 & 751 & 0.92 & 3.63 \\
CMK-3-PDA-1.1-10 & 771 & 0.93 & 3.47 \\
CMK-3-PDA-2.2-10 & 640 & 0.73 & 3.47 \\
CMK-3-PDA-4.4-10 & 415 & 0.47 & 3.32 \\
CMK-3-PDA-1.1-5 & 799 & 0.97 & 3.63 \\
CMK-3-PDA-1.1-24 & 700 & 0.77 & 3.47 \\
\hline
\end{tabular}


Table S3. Elemental composition, functional group densities and U(VI) adsorption capacities of PDA coated CMK-3.

\begin{tabular}{|c|c|c|c|c|c|c|c|c|c|}
\hline \multirow{2}{*}{ Samples } & \multicolumn{3}{|c|}{$\begin{array}{l}\text { element composition } \\
\text { (wt \%) }\end{array}$} & \multicolumn{2}{|c|}{$\begin{array}{c}-\mathrm{NH} \\
\text { density }\end{array}$} & \multicolumn{2}{|c|}{$-\mathrm{OH}$} & \multirow{2}{*}{$\begin{array}{c}\mathrm{Q} \\
\mathrm{mg} / \mathrm{g}\end{array}$} & \multirow{2}{*}{$\frac{K_{\mathrm{d}}}{\mathrm{cm}^{3} / \mathrm{g}}$} \\
\hline & $\mathrm{C}$ & $\mathrm{H}$ & $\mathrm{N}$ & $\begin{array}{c}\mu \mathrm{mol} / \\
\mathrm{m}^{2}\end{array}$ & $\begin{array}{l}\text { group/ } \\
\mathrm{nm}^{2}\end{array}$ & $\begin{array}{l}\mu \mathrm{mo} \\
1 / \mathrm{m}^{2}\end{array}$ & $\begin{array}{l}\text { group/ } \\
\mathrm{nm}^{2}\end{array}$ & & \\
\hline CMK-3 & 91.4 & 1.1 & 0.2 & 0 & 0 & 0 & 0 & 25.1 & 548 \\
\hline CMK-3-PDA-0.6-10 & 89.2 & 1.7 & 1.0 & 0.8 & 0.5 & 1.5 & 0.9 & 43.2 & 1045 \\
\hline CMK-3-PDA-1.1-10 & 87.7 & 1.8 & 1.5 & 1.2 & 0.7 & 2.4 & 1.4 & 53.7 & 1388 \\
\hline CMK-3-PDA-2.2-10 & 84.9 & 2.0 & 2.1 & 2.1 & 1.3 & 4.2 & 2.5 & 75.0 & 2245 \\
\hline CMK-3-PDA-4.4-10 & 80.9 & 2.4 & 2.9 & 4.7 & 2.8 & 9.3 & 5.6 & 93.6 & 3286 \\
\hline CMK-3-PDA-1.1-5 & 90.2 & 1.5 & 1.1 & 0.8 & 0.5 & 1.6 & 1.0 & 44.8 & 1101 \\
\hline CMK-3-PDA-1.1-24 & 85.1 & 1.8 & 2.1 & 2.0 & 1.2 & 3.9 & 2.3 & 76.3 & 2321 \\
\hline
\end{tabular}

